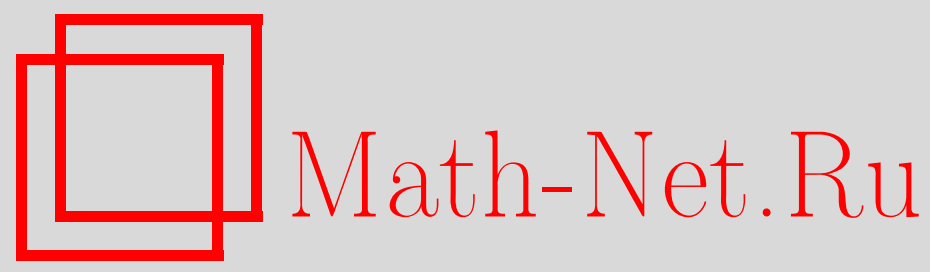

Г. В. Гришина, О компактности носителя решений нелинейных эллиптических и параболических уравнений второго порядка в полуцилиндре, Матем. заметки, 1997, том 62, выпуск 5, 700-711

DOI: https://doi.org/10.4213/mzm1657

Использование Общероссийского математического портала Math-Net.Ru подразумевает, что вы прочитали и согласны с пользовательским соглашением http://www. mathnet.ru/rus/agreement

Параметры загрузки:

IP : 34.229 .108 .108

26 апреля 2023 г., 14:06:35

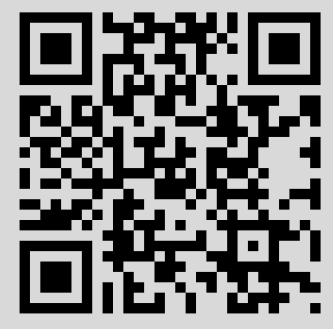


том 62 выпУск 5 нояьрь 1997

УДК 517.956

\section{О КОМПАКТНОСТИ НОСИТЕЛЯ РЕШЕНИЙ НЕЛИНЕЙНЫХ ЭЛЛИПТИЧЕСКИХ И ПАРАБОЛИЧЕСКИХ УРАВНЕНИЙ ВТОРОГО ПОРЯДКА В ПОЛУЦИЛИНДРЕ}

\section{Г.В. Гришина}

В работе изучаются решения уравнений вида

$$
\begin{gathered}
u_{t t}+L u+b(x, t) u_{t}=a(x, t)|u|^{\sigma-1} u \\
-u_{t}+L u=a(x, t)|u|^{\sigma-1} u
\end{gathered}
$$

где $L$ - равномерно эллиптический оператор. Рассмотрен случай $0<\sigma<1$. Найдены условия, при которых решения уравнения в полуцилиндре $\Pi_{0, \infty}=\{(x, t): x=$ $\left.\left(x_{1}, \ldots, x_{n}\right) \in \Omega, t>0\right\}$, где $\Omega \subset \mathbb{R}^{n}$ - ограниченная область, удовлетворяющие при $x \in \partial \Omega, t>0$ однородному условию Неймана, имеют компактный носитель. Устанавливаются утверждения типа: если $u(x, t)=o\left(t^{\gamma}\right)$ при $t \rightarrow \infty$, то существует $T$ такое, что при $t>T u(x, t) \equiv 0$. При этом $\gamma$ зависит от коэффициентов уравнения и степенного показателя $\sigma$.

Библиографоия: 15 названий.

В полуограниченном цилиндре $\Pi_{0, \infty}=\left\{(x, t): x=\left(x_{1}, \ldots, x_{n}\right) \in \Omega, t>0\right\}$, где $\Omega \subset \mathbb{R}^{n}$ - ограниченная область с гранищей $\partial \Omega \in C^{1,1}$, будем рассматривать уравнения вида

$$
\begin{aligned}
u_{t t}+ & L u+b(x, t) u_{t}=a(x, t)|u|^{\sigma-1} u \\
u_{t t}+ & \mathscr{L} u+b(x, t) u_{t}=a(x, t)|u|^{\sigma-1} u \\
& -u_{t}+L u=a(x, t)|u|^{\sigma-1} u \\
& -u_{t}+\mathscr{L} u=a(x, t)|u|^{\sigma-1} u
\end{aligned}
$$

где $0<\sigma<1$,

$$
\begin{gathered}
L u=\sum_{i, j=1}^{n} a_{i j}(x, t) u_{x_{i} x_{j}}+\sum_{i=1}^{n} a_{i}(x, t) u_{x_{i}} \\
\mathscr{L} u=\sum_{i, j=1}^{n}\left(a_{i j}(x, t) u_{x_{j}}\right)_{x_{i}}+\sum_{i=1}^{n} a_{i}(x, t) u_{x_{i}} .
\end{gathered}
$$

Работа выполнена при частичной поддержке Российского фонда фундаментальных исследований, грант № 96-01-00504. 
- равномерно эллиптические операторы такие, что для любых $(x, t) \in \Pi_{0, \infty}$ и $\xi=$ $\left(\xi_{1}, \ldots, \xi_{n}\right) \in \mathbb{R}^{n}$

$$
\lambda|\xi|^{2} \leqslant \sum_{i, j=1}^{n} a_{i j}(x, t) \xi_{i} \xi_{j} \leqslant \Lambda|\xi|^{2}, \quad \lambda, \Lambda>0 .
$$

Коэффициенты $a_{i j}(x, t)=a_{j i}(x, t), a_{i}(x, t), a(x, t), b(x, t)$ - ограниченные, измеримые на любом конечном цилиндре $\Pi_{0, t_{0}}=\left\{(x, t): x \in \Omega, t \in\left(0, t_{0}\right)\right\}, t_{0}>1$, функции, $a(x, t) \geqslant a_{0}(t+1)^{-\varepsilon}>0,|b(x, t)| \leqslant b_{0}(t+1)^{\alpha}, a_{0}, b_{0}=$ const $>0, \alpha, \varepsilon \in \mathbb{R}$. В операторе $L a_{i}(x, t) \in L_{q}\left(\Pi_{0, t_{0}}\right), q>n$.

Предполагаем, что на боковой границе цилиндра задано однородное условие Неймана

$$
\sum_{i, j=1}^{n} a_{i j}(x, t) u_{x_{j}} \nu_{i}=0, \quad x \in \partial \Omega
$$

где $\nu$ - вектор единичной внешней нормали к $\partial \Omega$.

Уравнения (1), (2) при $\sigma>1$ и $0<\sigma<1$ имеют различные свойства. Как известно, при $\sigma>1$ всякое решение уравнения

$$
\Delta u+k u_{t}=|u|^{\sigma-1} u
$$

удовлетворяющее условию (5), стремится к 0 при $t \rightarrow \infty$ [1]. При этом характер стремления к 0 зависит от знака $k$, а именно:

$$
u= \begin{cases}O\left(t^{1 /(1-\sigma)}\right) & \text { при } k<0, \\ O\left(t^{2 /(1-\sigma)}\right) & \text { при } k=0, \\ O\left(e^{-\mu t}\right) & \text { при } k>0 .\end{cases}
$$

(cм. [2]).

Если $0<\sigma<1$, то уравнение (6) может иметь как растущие при $t \rightarrow \infty$, так и имеющие компактный носитель решения. Например, при $k=0$ это будут функции

$$
\begin{gathered}
u_{1}=\left[\frac{(1-\sigma)^{2}}{2(1+\sigma)}\right]^{1 /(1-\sigma)} t^{2 /(1-\sigma)}, \\
u_{2}= \begin{cases}{\left[\frac{(1-\sigma)^{2}}{2(1+\sigma)}\right]^{1 /(1-\sigma)}(T-t)^{2 /(1-\sigma)},} & 1<t<T, \\
0, & t \geqslant T .\end{cases}
\end{gathered}
$$

В связи с этим возникает вопрос о предельном порядке роста решения $u(x, t)=o\left(t^{\gamma}\right)$ при $t \rightarrow \infty$, для которого можно утверждать, что решение имеет компактньй носитель. Из настоящей работы следует, в частности, что для $0<\sigma<1$ поведение решения уравнения (6) при $t \rightarrow \infty$ в отличии от случая $\sigma>1$ не зависит от знака $k$. А именно, при любом $k$, если $u(x, t)=o\left(t^{2 /(1-\sigma)}\right)$, то существует $T>1$, для которого $u \equiv 0$ при $t \geqslant T$. Это означает, что такие уравнения не могут иметь знакопостоянных или осциллирующих решений, стремящихся к 0 при $t \rightarrow \infty$. 
Как будет показано, наличие в уравнениях (1) и (2) членов, содержаших $u_{t}$, и поведение при $t \rightarrow \infty$ коэффициентов $a(x, t), b(x, t)$ существенно влияют на условия, при которых решения задач $(1),(5) ;(2),(5) ;(3),(5) ;(4),(5)$ имеют компактньй носитель.

Вопрос о "мертвых зонах" для уравнений типа $(1),(2)$ при $0<\sigma<1$ рассматривался ранее в работах [3]-[10] при более жестких условиях на коэффициенты. В частности, везде существенно используется отделенность от нуля коэффициента при нелинейном члене уравнения. Обрашение этого коэффициента в 0 на множестве положительной меры допускается в работе [11], но при этом изучаются только задачи $(2),(5)$ и $(4),(5)$ для уравнений, не содержащих младших членов, с коэффициентами, не зависящими от $t$. Эффект локализации решений полулинейных параболических уравнений изучался также в работах [12], [13].

Рассуждения настоящей работы основаны на рассмотрении решений соответствующих обыкновенных дифференциальных уравнений

$$
\begin{gathered}
y^{\prime \prime} \pm b(t+1)^{\alpha} y^{\prime}=a(t+1)^{-\varepsilon}|y|^{\sigma-1} y \\
-y^{\prime}=a(t+1)^{-\varepsilon}|y|^{\sigma-1} y
\end{gathered}
$$

и применении принципа максимума.

Автор благодарит профессора В. А. Кондратьева за полезные обсуждения.

\section{1. Эллиптические уравнения.}

ОПРЕДЕЛЕНИЕ 1. Классическим решением задачи (1), (5) называется функция $u(x, t) \in C^{2}\left(\Pi_{0, \infty}\right) \cap C^{1}\left(\overline{\Pi_{0, \infty}}\right)$, удовлетворяющая в $\Pi_{0, \infty}$ уравнению (1) и условию (5).

ОПРЕДЕЛЕНИЕ 2. Слабым решением задачи $(2),(5)$ называется функция $u(x, t) \in$ $W_{2}^{1}\left(\Pi_{0, t_{0}}\right), t_{0}>0$, такая, что для любой функции $\xi(x, t) \in W_{2}^{1}\left(\Pi_{0, t_{0}}\right)$, удовлетворяющей условиям $\xi(x, t) \geqslant 0, \xi(x, 0) \equiv 0, \xi(x, t) \equiv 0$ при $t \geqslant t_{0}, x \in \bar{\Omega}$, справедливо тождество

$$
Q(x, t)=\int_{\Pi_{0, t_{0}}} a(x, t)|u|^{\sigma-1} u \xi d x d t
$$

где

$$
Q(x, t)=\int_{\Pi_{0, t_{0}}}\left[-u_{t} \xi_{t}-\sum_{i, j=1}^{n} a_{i j}(x, t) u_{x_{j}} \xi_{x_{i}}+\sum_{i, j=1}^{n} a_{i}(x, t) u_{x_{i}} \xi+b(x, t) u_{t} \xi\right] d x d t .
$$

Теорема 1. Пусть в уравнении $(1) \quad b(x, t) \geqslant 0, \varepsilon \leqslant 2, \alpha-$ произвольное действительное число и классическое решение задачи (1), (5) $u(x, t) \rightarrow 0$ при $t \rightarrow \infty$. Тогда найдется $T>0$ такое, что $u(x, t) \equiv 0$ при $t>T$.

ЗАмЕчАниЕ 1 . Условие $\varepsilon \leqslant 2$ точное. При $\varepsilon>2$ уравнение

$$
\Delta u=(t+1)^{-\varepsilon}|u|^{\sigma-1} u
$$

имеет два знакоопределенных, стремяшихся к 0 при $t \rightarrow \infty$ решения

$$
u_{1,2}= \pm\left[\frac{(1-\sigma)^{2}}{(1+\sigma-\varepsilon)(2-\varepsilon)}\right]^{1 /(1-\sigma)}(t+1)^{(2-\varepsilon) /(1-\sigma)}
$$


удовлетворяюших на боковой поверхности цилиндра однородному условию Неймана.

Тем не менее, утверждение теоремы справедливо и при $\varepsilon>2$, если дополнительно предположить, что $\max _{x \in \bar{\Omega}}|u(x, 0)|$ достаточно мал, или $u(x, t) \rightarrow 0$ с указанной скоростью.

ДоКАЗАТЕЛЬСТВо ТЕОРЕМЫ 1. Рассмотрим функцию

$$
v(t)= \begin{cases}C(T-t)^{2 /(1-\sigma)}, & 0<t<T \\ 0, & t \geqslant T\end{cases}
$$

Очевидно, $v(t) \in C^{2}\left(\overline{\Pi_{0, \infty}}\right)$ и удовлетворяет краевому условию (5). Выбирая постоянную $C$ так, чтобы для любого $t \in(0, T)$ вьполнялось неравенство

$$
v_{t t}+L v+b(x, t) v_{t}-a(x, t)|v|^{\sigma-1} v \leqslant 0
$$

получим

$$
C \leqslant \begin{cases}{\left[\frac{a_{0}(1-\sigma)^{2}}{2(1+\sigma)}\right]^{1 /(1-\sigma)},} & \varepsilon \leqslant 0, \\ {\left[\frac{a_{0}(1-\sigma)^{2}}{2(T+1)^{\varepsilon}(1+\sigma)}\right]^{1 /(1-\sigma)},} & \varepsilon>0 .\end{cases}
$$

Теперь, зафиксировав $C$, определим $T$ из условия

$$
M=\max _{x \in \bar{\Omega}}|u(x, 0)|<v(0)=C T^{2 /(1-\sigma)} .
$$

Учитьвая оценки для $C$, получим

$$
M< \begin{cases}{\left[\frac{a_{0}(1-\sigma)^{2}}{2(1+\sigma)}\right]^{1 /(1-\sigma)} T^{2 /(1-\sigma)},} & \varepsilon \leqslant 0 \\ {\left[\frac{a_{0}(1-\sigma)^{2}}{2(T+1)^{\varepsilon}(1+\sigma)}\right]^{1 /(1-\sigma)} T^{2 /(1-\sigma)},} & \varepsilon>0 .\end{cases}
$$

Первое неравенство всегда разрешимо относительно $T$, а второе разрешимо при любом $M$ только для $\varepsilon<2$. Если $\varepsilon=2$, правая часть второго неравенства в (9) монотонно возрастает при $T \rightarrow \infty$, причем

$$
\lim _{T \rightarrow \infty}\left[\frac{(1-\sigma)^{2}}{2(T+1)^{2}(1+\sigma)}\right]^{1 /(1-\sigma)} T^{2 /(1-\sigma)}=\left[\frac{a_{0}(1-\sigma)}{2(1+\sigma)}\right]^{1 /(1-\sigma)}=M_{a} .
$$

Следовательно, если $M<M_{a}$, то $T$ может быть найдено.

Пусть теперь $\varepsilon=2, M \geqslant M_{a}$. В этом случае рассмотрим функцию $v(t)$ при $t \geqslant T_{0}>0$. Тогда $T$ нужно выбирать из условия

$$
M_{T_{0}}=\max _{x \in \bar{\Omega}}\left|u\left(x, T_{0}\right)\right|<v\left(T_{0}\right)=C\left(T-T_{0}\right)^{2 /(1-\sigma)}<M_{a}\left[\frac{T-T_{0}}{T+1}\right]^{2 /(1-\sigma)} .
$$


Так как

$$
\lim _{T \rightarrow \infty}\left[\frac{T-T_{0}}{T+1}\right]^{2 /(1-\sigma)}=1 \quad \forall T_{0} \geqslant 0
$$

и $u(x, t) \rightarrow 0$ при $t \rightarrow \infty$, найдется $T_{0} \geqslant 0$ такое, что $M_{T_{0}}<M_{a}$, а значит, можно подобрать $T>T_{0}$, при котором справедливо неравенство (10).

Итак, пусть выбраны $C, T_{0} \geqslant 0, T$. Тогда для функции $v$ вьполняется неравенство (8). Рассмотрим функцию $w(x, t)=v(t)-u(x, t)$. При $t=T_{0} \geqslant 0$ функция $w>0$ и $w \rightarrow 0$ при $t \rightarrow \infty$. Покажем, что $w \geqslant 0$ в $\Pi_{T_{0}, \infty}$. Если предположить, что последнее неравенство нарушается, то функция $w$ должна иметь отрицательньй минимум

$$
\min _{\Pi_{T_{0}, \infty}} w(x, t)=-\delta
$$

Так как $w \rightarrow 0$ при $t \rightarrow \infty$, найдется $T_{1}>T$ такое, что $|w| \leqslant \delta / 2$ при $t \geqslant T_{1}$, но тогда

$$
\min _{\Pi_{T_{0}, T_{1}}} w(x, t)=-\delta
$$

Заметим, что минимум не может достигаться при $t=T_{0}$ и $t=T_{1}$. Кроме того, учитывая (8), имеем

$$
w_{t t}+L w+b(x, t) w_{t} \leqslant a(x, t)\left(|v|^{\sigma-1} v-|u|^{\sigma-1} u\right) .
$$

Если $u \neq v$, то правая часть этого неравенства имеет тот же знак, что и $w$. Поэтому из принципа максимума [14] следует, что отрицательньй минимум не может достигаться ни внутри цилиндра $\Pi_{T_{0}}, T_{1}$, ни на его боковой поверхности.

Таким образом, $w \geqslant 0$ в $\Pi_{T_{0}, T_{1}}$. Устремляя $T_{1}$ к бесконечности, получим, что $v \geqslant u$ в $\Pi_{T_{0}, \infty}$. Проводя аналогичные рассуждения для $-u$, получим, что $u \geqslant-v$. Но $v \equiv 0$ при $t \geqslant T$, а следовательно, $u \equiv 0$ при $t \geqslant T$.

Теорема доказана.

Пусть теперь $\varepsilon>2$. Тогда правая часть неравенства (9) принимает максимальное значение при $T=2 /(\varepsilon-2)>1$. Значит, подходящее $T$ может быть найдено, если

$$
M<\left[\frac{a_{0}(1-\sigma)^{2}(\varepsilon-2)^{\varepsilon-2}}{2(1+\sigma) \varepsilon^{\varepsilon}}\right]^{1 /(1-\sigma)} .
$$

В противном случае рассмотрим при $\varepsilon>2$ функцию $v(t)$ при $t \geqslant T_{0}=T / 2>0$ и придем к необходимости выполнения условия

$$
M_{T_{0}}<M_{a}\left[\frac{(\varepsilon-2)^{\varepsilon-2}}{\varepsilon^{\varepsilon}}\right]^{1 /(1-\sigma)}\left(2 T_{0}+1\right)^{(2-\varepsilon) /(1-\sigma)} .
$$

Правая часть этого неравенства стремится к 0 при $\varepsilon>2, T_{0} \rightarrow \infty$. Следовательно, оно может быть вьполнено при некотором $T_{0}$, если, например, задана скорость стремления к 0 решения $u(x, t)$ при $t \rightarrow \infty$. Это может быть условие типа

$$
u(x, t)=o\left(t^{(2-\varepsilon) /(1-\sigma)}\right), \quad t \rightarrow \infty .
$$

Итак, справедлива 
Теорема 2. Пусть в уравнении $(1) \quad b(x, t) \geqslant 0, \varepsilon>2, \alpha-$ произвольное действительное число, $u(x, t)$ - классическое решение задачи (1), (5). Тогда, если

$$
\max _{x \in \bar{\Omega}}|u(x, 0)|<\left[\frac{a_{0}(1-\sigma)^{2}(\varepsilon-2)^{\varepsilon-2}}{2(1+\sigma) \varepsilon^{\varepsilon}}\right]^{1 /(1-\sigma)}, \quad u(x, t) \rightarrow 0, \quad t \rightarrow \infty
$$

или выполнено (11), то найдется $T>0$ такое, что $u(x, t) \equiv 0$ при $t \geqslant T$.

Рассмотрим теперь задачу $(2),(5)$ в $\Pi_{0, \infty}$. Всюду в дальнейшем, где речь идет о слабых решениях, можно считать, что $\partial \Omega \in C^{0,1}$.

Заметим, что если функция $v(t)$ удовлетворяет неравенству $(8)$ п.в. в $\Pi_{0, \infty}$ и имеет непрерьвную первую производную, то для нее это неравенство справедливо и в слабом смысле, т.е.

$$
Q(x, t) \leqslant \int_{\Pi_{0, \infty}} a(x, t)|v|^{\sigma-1} v \xi d x d t
$$

для любой $\xi(x, t)$, описанной в определении 2. Таким образом, повторяя для слабых решений задачи (2), (5), стремящихся к 0 при $t \rightarrow \infty$, рассуждения, приведенные в доказательствах теорем 1 и 2 , придем к рассмотрению функции $w$, для которой справедлива оценка

$$
Q(x, t) \leqslant \int_{\Pi_{0, \infty}} a(x, t)\left(|v|^{\sigma-1} v-|u|^{\sigma-1} u\right) \xi d x d t .
$$

По принципу максимума для уравнений в дивергентной форме [8] $w$ не может иметь отрицательного минимума во внутренних точках $\Pi_{0, \infty}$. Очевидно, он не может достигаться при $t=0$. Покажем, что никакая точка $\left(x_{0}, t_{0}\right)$ на боковой поверхности $\Pi_{0, \infty}$ не может быть точкой отрицательного минимума функции $w$. Действительно, после локального распрямления границы цилиндра в окрестности точки $\left(x_{0}, t_{0}\right)$ с помощью липшищева преобразования и продолжения решения $u(x, t)$ уравнения $(2)$ через распрямленный участок гранищы с учетом условия (5) четным образом получим функцию, принадлежащую в окрестности $\left(x_{0}, t_{0}\right)$ тому же классу, что и $u(x, t)$, и удовлетворяющую уравнению той же структуры, что и $(2)$, но точка $\left(x_{0}, t_{0}\right)$ уже будет внутренней для этой окрестности. Свойство точки $\left(x_{0}, t_{0}\right)$ быть точкой минимума для $u(x, t)$ сохраняется и для продолженной четным образом функции. При этом остается верным и неравенство (12) для продолженной через границу функции $w$. Применяя принцип максимума, приходим к противоречию.

Итак, утверждения теорем 1 и 2 имеют место и для слабых решений задачи (2), (5), стремящихся к 0 при $t \rightarrow \infty$.

ЗАмЕчАниЕ 2. Значение параметра $\varepsilon=2$ является критическим при рассмотрении стремяшихся к 0 при $t \rightarrow \infty$ решений. В то же время, зависимость от $t$ коэффициента при нелинейном члене уравнения существенно влияет на поведение решения при $t \rightarrow \infty$. Действительно, в работе [11] показано, что если решение уравнения

$$
u_{t t}+\sum_{i, j=1}^{n}\left(a_{i j}(x) u_{x_{j}}\right)_{x_{i}}=a(x)|u|^{\sigma-1} u
$$


в $\Pi_{0, \infty}$, удовлетворяющее условию $(5)$, где $a(x) \geqslant 0$, таково, что

$$
\lim _{t \rightarrow \infty} t^{-1}\|u(x, t)\|_{L_{1}(\Omega)}=0
$$

то существуют $C, \mu>0$, для которых при достаточно больших $t$ справедлива оценка $|u(x, t)| \leqslant C e^{-\mu t}$. Если же $\alpha(x) \geqslant a_{0}>0$, то такие решения имеют компактный носитель.

Из доказанных теорем следует, что уравнение

$$
\Delta u=(1+t)^{-\varepsilon}|u|^{\sigma-1} u
$$

вообще не имеет экспоненциально убьвающих решений, а условия на рост решения при $t \rightarrow \infty$, достаточные для компактности носителя, зависят от $\varepsilon$.

Теорема 3. Пусть в уравнениях (2) $u(1) b(x, t) \geqslant-B_{1}(t+1)^{\alpha}$, где $B_{1}>0$, $u(x, t)$ - решение задачи (1), (5) или (2), (5) в $\Pi_{0, \infty}$, причем $u(x, t) \rightarrow 0$ при $t \rightarrow \infty$. Тогда найдется $T>0$ такое, что $u(x, t) \equiv 0$ при $t \geqslant T$, если выполнено одно из следующих условий:

1) $\varepsilon \leqslant 0, \alpha<1$

2) $0 \leqslant \varepsilon \leqslant 1, \alpha \leqslant 1-\varepsilon$

3) $\varepsilon \leqslant 0, \alpha>1, u(x, t)=o\left(t^{(1-\alpha) /(1-\sigma)}\right), t \rightarrow \infty$;

4) $\varepsilon>1, \alpha \leqslant 0, u(x, t)=o\left(t^{(1-\varepsilon) /(1-\sigma)}\right), t \rightarrow \infty$;

5) $\varepsilon>0, \alpha>\max \{0,1-\varepsilon\}, u(x, t)=o\left(t^{(1-\alpha-\varepsilon) /(1-\sigma)}\right), t \rightarrow \infty$.

Доказательство этой теоремы также основано на построении барьерной функции

$$
v(t)= \begin{cases}C(T-t)^{2 /(1-\sigma)}, & t \in\left(T_{0}, T\right), \\ 0, & t \geqslant T\end{cases}
$$

удовлетворяющей неравенству (8), и применении принципа максимума. Отличие состоит в том, что постоянную $C$ необходимо выбирать согласно оценке

$$
C \leqslant\left[\frac{1}{a}(t+1)^{\varepsilon}\left(\frac{2(1+\sigma)}{(1-\sigma)^{2}}+\frac{2 B_{1}}{1-\sigma}(t+1)^{\alpha}(T-t)\right)\right]^{1 /(\sigma-1)}
$$

которая должна быть справедлива для всех $t \in(0, T)$.

ЗАмЕчАнИЕ 3 . Как и в теореме 2 , можно указать постоянные $M_{1}, M_{2}, M_{3}$, для которых из условия $\max _{x \in \bar{\Omega}}|u(x, t)| \leqslant M_{i}, i=1,2,3$, если $u(x, t) \rightarrow 0$ при $t \rightarrow \infty$, следует, что решение имеет компактный носитель, когда выполнены соотношения между $\varepsilon$ и $\alpha$ из условий 3)-5) соответственно.

ТЕОРема 4. Пусть $u(x, t)$ - решение задачи (1), (5) или (2), (5) в $\Pi_{0, \infty}$, причем $-B_{1}(T+1)^{\alpha} \leqslant b(x, t) \leqslant B_{2}(T+1)^{\alpha}$, әде $B_{1}, B_{2} \geqslant 0$. Тогда найдется $T>0$ такое, что $u(x, t) \equiv 0$ при $t \geqslant T$, если

1) $u(x, t)=o\left(t^{(2-\varepsilon) /(1-\sigma)}\right), t \rightarrow \infty, \varepsilon \leqslant 2 \sigma$ в случае задачи (1), (5) или $\varepsilon \leqslant 1+\sigma$ в случае задачи (2), (5), и выполнено одно из следуюших условий:

a) $B_{1}=0, B_{2}=0$; 
б) $B_{1}>0, B_{2}>0, \alpha \leqslant-1, \varepsilon \leqslant 1$;

в) $B_{1}=0, B_{2}>0, \alpha \leqslant-1$;

г) $B_{1}>0, B_{2}=0, \varepsilon \leqslant 0, \alpha \leqslant 1$ или $0 \leqslant \varepsilon \leqslant 1, \alpha \leqslant 1-\varepsilon$; uлu

2) $u=o\left(t^{(1-\alpha-\varepsilon) /(1-\sigma)}\right), t \rightarrow \infty, 1+\alpha+\varepsilon \leqslant 2 \sigma$ в случае задачи (1), (5) или $\alpha+\varepsilon \leqslant \sigma$ в случае задачи (2), (5), и выполнено одно из следующих условий:

д) $B_{1}=0, B_{2}>0, \alpha \geqslant-1$;

e) $B_{1}>0, B_{2}>0,-1 \leqslant \alpha \leqslant 1$.

ЗАмЕчание 4. Условие $B_{2}>0$ имеет смысл, когда $\operatorname{mes}\left\{(x, t) \in \Pi_{0, \infty}: b(x, t)>0\right\}>0$ для всех $t_{0}>0$. В противном случае можно считать, что $B_{2}=0$.

ЗАмечАниЕ 5. Условия $\varepsilon \leqslant 2 \sigma$ или $\varepsilon \leqslant 1+\sigma$ и $1+\alpha+\varepsilon \leqslant 2 \sigma$ или $\alpha+\varepsilon \leqslant \sigma$ продиктованы методом доказательства.

ДоКАЗАТЕЛЬСТво ТЕОРЕмЫ 4 . Покажем, что $u(x, t) \rightarrow 0$ при $t \rightarrow \infty$. Предположим, что это не так. Тогда найдется $\delta>0$ и последовательность $\left\{T_{k}\right\} \rightarrow \infty, k \rightarrow \infty$, такая, что

$$
\max _{x \in \bar{\Omega}}\left|u\left(x, T_{k}\right)\right|>2 \delta>0 \quad \forall k \geqslant k_{0} .
$$

Пусть для определенности

$$
\max _{x \in \bar{\Omega}}\left|u\left(x, T_{k}\right)\right|=u\left(x^{(k)}, T_{k}\right)>2 \delta,
$$

где $x^{(k)} \in \Omega$. Построим семейство функций

$$
v_{k}(t)= \begin{cases}C_{k}^{(1)}\left[\left(T_{k}-t\right)^{2 /(1-\sigma)}+\delta_{1}\right], & 1<t<T_{k}, \\ C_{k}^{(2)}\left[\left(T_{k}-t\right)^{\gamma}+\delta_{1}\right], & t>T_{k},\end{cases}
$$

где $\gamma=(2-\varepsilon) /(1-\sigma)$ или $\gamma=(1-\alpha-\varepsilon) /(1-\sigma)$, если выполнены условия 1) или 2) соответственно, а

$$
\delta_{1}= \begin{cases}0 & \text { при } \varepsilon \leqslant 0 \\ \delta & \text { при } \varepsilon>0 .\end{cases}
$$

При $\delta_{1}=0$ постоянные $C_{k}^{(1)}$ и $C_{k}^{(2)}$ могут быть выбраны независимо друг от друга. Постоянная $C_{k}^{(1)}$ выбирается так, чтобы

$$
\max _{x \in \bar{\Omega}}|u(x, 0)|<v_{k}(0)=C_{k}^{(1)}\left[T_{k}^{2 /(1-\sigma)}+\delta_{1}\right]
$$

и выполнялось неравенство (8). Из доказательств теорем 1 и 2 следует, что при $\varepsilon \leqslant 0$ неравенство (8) для $v_{k}(t)$ на $\left(0, T_{k}\right)$ эквивалентно условию $C_{k}^{(1)} \leqslant C=$ const, а при $\varepsilon>0$ - условию $C_{k}^{(1)} \leqslant C_{1}\left(T_{k}\right)$, где $C_{1}\left(T_{k}\right) \rightarrow 0$ при $k \rightarrow \infty$. Оценка (13) накладывает ограничения на скорость стремления к 0 последовательности $C_{1}\left(T_{k}\right)$ при $k \rightarrow \infty$. Поэтому выберем $C_{k}^{(1)}$ из условий

$$
\rho_{1} C_{1}\left(T_{k}\right) \leqslant C_{k}^{(1)} \leqslant \begin{cases}\min \{1, C\}, & \varepsilon \leqslant 0, \\ \min \left\{1, C_{1}\left(T_{k}\right)\right\}, & \varepsilon>0\end{cases}
$$


где $\rho_{1}=$ const $>0$. Неравенство $(8)$ для $v_{k}(t)$ при $t \geqslant T_{0}$ дает оценку

$$
C_{k}^{(2)} \leqslant\left[\frac{2^{\sigma-1} a_{0}\left[\left(t-T_{k}\right)^{\gamma \sigma}+\delta_{1}^{\sigma}\right]}{\gamma(t+1)^{\varepsilon}\left(t-T_{k}\right)^{\gamma-2}\left[\gamma-1+B_{2}(t+1)^{\alpha}\left(t-T_{k}\right)\right]}\right]^{1 /(1-\sigma)}, \quad t \geqslant T_{k} .
$$

Анализируя эту оценку, получим, чтопри $\varepsilon \leqslant 0 C_{k}^{(2)} \leqslant C_{0}$, где $C_{0}=$ const, не зависящая от $k$, а при $\varepsilon>0 C_{k}^{(2)} \leqslant C^{(2)}\left(T_{k}, \delta_{1}\right)$, где $\rho_{2} C_{1}\left(T_{k}\right) \leqslant C^{(2)}\left(T_{k}, \delta_{1}\right), \rho_{2}=$ const $>0$. Поскольку других ограничений на $C_{k}^{(2)}$ нет, можно взять $C_{k}=C_{k}^{(1)}=C_{k}^{(2)}$ так, что

$$
\min \left\{\rho_{1}, \rho_{2}\right\} C_{1}\left(T_{k}\right) \leqslant C_{k} \leqslant \begin{cases}\min \left\{1, C, C_{0}\right\}, & \varepsilon \leqslant 0 \\ \min \left\{1, C_{1}\left(T_{k}\right), \rho_{2} C_{1}\left(T_{k}\right)\right\}, & \varepsilon>0\end{cases}
$$

Рассмотрим теперь функцию $w_{k}(x, t)=v_{k}(t)-u(x, t)$. Из сказанного вьше следует, что $w_{k}(x, 0)>0, w_{k}\left(x^{(k)}, T_{k}\right)<0, w_{k}(x, t) \rightarrow+\infty$ при $t \rightarrow \infty$, и следовательно, в некотором цилиндре $\Pi_{0, T_{k}^{\prime}}$, где $T_{k}^{\prime}>T_{k}$, функция $w_{k}(x, t)$ имеет отрицательньй минимум. Но это противоречит принципу максимума. Значит, $u(x, t) \rightarrow 0$ при $t \rightarrow \infty$. Тогда утверждение теоремы следует из теорем 1 и 3.

ЗАмЕчАниЕ 6 . Из доказательства следует, что размер носителя (величина $T$ ) определяется максимумом модуля решения при $t=0$.

Суммируя полученные результаты, сформулируем некоторые следствия из них.

СлЕДСТВИЕ 1. Пусть $и(x, t)$ - решение в $\Pi_{0, \infty}$ уравнения $(7)$, удовлетворяющее условию (5). Если выполнено условие

1) $\varepsilon \leqslant 1+\sigma$ uлu $\varepsilon>2, u(x, t)=o\left(t^{(2-\varepsilon) /(1-\sigma)}\right)$ npu $t \rightarrow \infty$, илu

2) $1+\sigma<\varepsilon \leqslant 2, u(x, t) \rightarrow 0$ npu $t \rightarrow \infty$,

то решение имеет компактный носитель.

СлЕДСТВИЕ 2. Пусть $u(x, t)-$ решение в $\Pi_{0, \infty}$ уравнения

$$
\Delta u+k u_{t}=(t+1)^{-\varepsilon}|u|^{\sigma-1} u, \quad k>0
$$

удовлетворяющее условию (5). Если выполнено одно из условий:

1) $\varepsilon \leqslant \sigma, u(x, t)=o\left(t^{(1-\varepsilon) /(1-\sigma)}\right), t \rightarrow \infty$,

2) $\sigma<\varepsilon \leqslant 2, u(x, t) \rightarrow 0, t \rightarrow \infty$,

3) $\varepsilon>2, u(x, t)=o\left(t^{(2-\varepsilon) /(1-\sigma)}\right), t \rightarrow \infty$,

то решение имеет компактный носитель.

СлЕДСТВИЕ 3. Пусть $u(x, t)$ - решение в $\Pi_{0, \infty}$ уравнения

$$
\Delta u-k u_{t}=(t+1)^{-\varepsilon}|u|^{\sigma-1} u, \quad k>0,
$$

удовлетворяющее условию (5). Если выполнено условие

1) $\varepsilon \leqslant 1, u(x, t)=o\left(t^{(2-\varepsilon) /(1-\sigma)}\right), t \rightarrow \infty$, uлu

2) $1<\varepsilon, u(x, t)=o\left(t^{(1-\varepsilon) /(1-\sigma)}\right), t \rightarrow \infty$, то решение имеет компактный носитель. 
ЗАмЕчАнИЕ 7 . При $\varepsilon=0$ решения уравнений (14) и (15) имеют компактный носитель, если $u(x, t)=o\left(t^{1 /(1-\sigma)}\right)$ и $u(x, t)=o\left(t^{2 /(1-\sigma)}\right)$ при $t \rightarrow \infty$ соответственно. $\mathrm{B}$ отличии от случая $\sigma>1$ оба уравнения не имеют знакоопределенных или осциллирующих решений таких, что $|u(x, t)| \leqslant C t$.

СлЕДСТВИЕ 4. Пусть $u(x, t)$ - решение в $\Pi_{0, \infty}$ уравнения

$$
\Delta u+k(t+1)^{\alpha} u_{t}=|u|^{\sigma-1} u, \quad k>0,
$$

удовлетворяющее условию (5). Если выполнено одно из условий:

1) $\alpha \leqslant-1, u(x, t)=o\left(t^{2 /(1-\sigma)}\right), t \rightarrow \infty$,

2) $-1<\alpha \leqslant \sigma, u(x, t)=o\left(t^{(1-\alpha) /(1-\sigma)}\right), t \rightarrow \infty$,

3) $\sigma<\alpha, u(x, t) \rightarrow 0, t \rightarrow \infty$,

то решение имеет компактный носитель.

СЛЕДСТВИЕ 5. Пусть $u(x, t)-$ решение в $\Pi_{0, \infty}$ уравнения

$$
\Delta u-k(t+1)^{\alpha} u_{t}=|u|^{\sigma-1} u, \quad k>0,
$$

удовлетворяющее условию (5). Если выполнено одно из условий:

1) $\alpha \leqslant 1, u(x, t)=o\left(t^{2 /(1-\sigma)}\right), t \rightarrow \infty$,

2) $\alpha>1, u(x, t)=o\left(t^{(1-\alpha) /(1-\sigma)}\right), t \rightarrow \infty$,

то решение имеет компактный носитель.

ЗАмЕчАниЕ 8. Условия на рост решения при $t \rightarrow \infty$ различаются для уравнений $(16)$ и (17), если $-1<\alpha \leqslant 1$. Например, уравнение (16) при $\alpha=\sigma$ имеет решение $u(x, t)=$ $\pm k^{1 /(\sigma-1)}(t+1)$, в то время, как всякое решение уравнения (17), удовлетворяющее условию $u(x, t)=o\left(t^{2 /(1-\sigma)}\right), 2 /(1-\sigma)>2$, имеет компактный носитель.

\section{2. Параболические уравнения.}

ОПРЕДЕЛЕНИЕ 3. Классическим решением задачи (3), (5) называется функция $u(x, t) \in C_{x, t}^{2,1}\left(\Pi_{0, \infty}\right) \cap C_{x, t}^{1,0}\left(\overline{\Pi_{0, \infty}}\right)$, удовлетворяющая в $\Pi_{0, \infty}$ уравнению (3) и условию (5).

ОПРЕДЕЛЕНИЕ 4. Слабым решением задачи (4), (5) называется функция $u(x, t) \in$ $W_{2}^{1}\left(\Pi_{0, t_{0}}\right), t_{0}>0$, такая, что для любой функции $\xi(x, t) \in W_{2}^{1}\left(\Pi_{0, t_{0}}\right)$, удовлетворяющей условиям $\xi(x, t) \geqslant 0, \xi(x, 0) \equiv 0, \xi(x, t) \equiv 0$ при $t \geqslant t_{0}, x \in \bar{\Omega}$, справедливо тождество

$$
\int_{\Pi_{0, t_{0}}}\left[-u \xi_{t}+\sum_{i, j=1}^{n} a_{i j}(x, t) u_{x_{j}} \xi_{x_{i}}-\sum_{i, j=1}^{n} a_{i}(x, t) u_{x_{i}} \xi+a(x, t)|u|^{\sigma-1} u \xi\right] d x d t=0
$$

Из принципа максимума [15] следует, что всякое решение задачи $(3),(5)$ или (4), (5) ограниченная функция.

Рассмотрим обыкновенное дифференциальное уравнение

$$
-y^{\prime}=a(t+1)^{-\varepsilon}|y|^{\sigma-1} y, \quad a>0 .
$$


В зависимости от $\varepsilon$ свойства решений различны. Так, например, при $\varepsilon \leqslant 1$ все решения имеют компактньй носитель. Это будут функции

$$
y(t)= \begin{cases} \pm\left[T-\frac{a(1-\sigma)}{1-\varepsilon}(t+1)^{1-\varepsilon}\right]^{1 /(1-\sigma)}, & 0<t<\frac{T(1-\varepsilon)}{a(1-\sigma)}-1 \\ 0, & t \geqslant \frac{T(1-\varepsilon)}{a(1-\sigma)}-1\end{cases}
$$

если $\varepsilon<1$, и

$$
y(t)= \begin{cases} \pm[T-a(1-\sigma) \ln (t+1)]^{1 /(1-\sigma)}, & 0<t<e^{T /(a(1-\sigma))}-1 \\ 0, & t \geqslant e^{T /(a(1-\sigma))}-1,\end{cases}
$$

при $\varepsilon=1$. При $\varepsilon>1$ можно указать знакопостоянные решения, стремящиеся к любому действительному числу при $t \rightarrow \infty$ :

$$
y(t)= \pm\left[\frac{a(1-\sigma)}{(\varepsilon-1)(t+1)^{\varepsilon-1}}+T\right]^{1 /(1-\sigma)} \rightarrow T^{1 /(1-\sigma)}, \quad t \rightarrow \infty
$$

В частности,

$$
y(t)= \pm\left[\frac{a(1-\sigma)}{\varepsilon-1}\right]^{1 /(1-\sigma)}(t+1)^{(1-\varepsilon) /(1-\sigma)} \rightarrow 0, \quad t \rightarrow \infty
$$

Используя в качестве барьеров функции

$$
v(t)= \begin{cases}C[T-\ln (t+1)]^{1 /(1-\sigma)}, & 0<t<e^{T}-1 \\ 0, & t \geqslant e^{T}-1\end{cases}
$$

при $\varepsilon=1$ и

$$
v(t)= \begin{cases}C(T-t)^{1 /(1-\varepsilon)}, & 0<t<T \\ 0, & t \geqslant T\end{cases}
$$

при $\varepsilon \neq 1$, получим следующую теорему.

ТЕОрема 5. Пусть $u(x, t)$ - решение задачи (3), (5) или (4), (5) в $\Pi_{0, \infty}$. Если $\varepsilon \leqslant 1$, то любое решение имеет компактный носитель. Если $\varepsilon>1 и$

$$
\max _{x \in \bar{\Omega}}|u(x, 0)| \leqslant\left[\frac{a_{0}(1-\sigma)(\varepsilon-1)^{\varepsilon-1}}{\varepsilon^{\varepsilon}}\right]^{1 /(1-\sigma)}
$$

или $u(x, t)=o\left(t^{(1-\varepsilon) /(1-\sigma)}\right)$ nри $t \rightarrow \infty$, то решение имеет компактный носитель. 


\section{СПИСОК ЦИТИРОВАННОЙ ЛИТЕРАТУРЫ}

[1] Kondratiev V. A., Oleinik O. A. On asymptotic behaviour of solutions of some nonlinear elliptic equations in unbounded domains // Partial Differential Equations and Related Subjects. Pitman Res. Notes Math. Ser. V. 269. Harlow: Longman Sci. Tech., 1992. P. 163-195.

[2] Кондратьев В. А., Олейник О. А. О поведении на бесконечности решений одного класса нелинейных эллиптических уравнений в цилиндрической области // Докл. РАН. 1995. Т. 341. № 4. C. 446-449.

[3] Diaz J. I. Solucionos con soporte compacto para ciertos problemas semilineares. Madrid: Editorial Univ. Complutense Madrid, 1976.

[4] Veron L. Equations d'évolution semi-linéaires du second ordre dans $L^{1} / /$ Rev. Roumaine Math. Pures Appl. 1982. V. 27. №1. P. 95-123.

[5] Diaz J. I. Nonlinear partial differential equations and free boundaries. 1. Pitman Res. Notes Math. Ser. V. 106. Harlow: Longman Sci. Tech., 1985.

[6] Кондратьев В.А., Ландис Е. М. О качественных свойствах решений одного нелинейного уравнения второго порядка // Матем. сб. 1988. Т. 135 (177). №3. С. 346-360.

[7] Landis E. M. Some properties of the solution of degenerating semilinear elliptic inequalities // Russian J. Math. Phys. 1993. V. 1. № 4. P. 483-494.

[8] Ландис Е. М. О "мертвой зоне" для полулинейных вырождающихся эллиптических неравенств // Дифференц. уравнения. 1993. Т. 29. №3. С. 414-423.

[9] Калашников А. С. О возмущении критических показателей в некоторых нелинейных задачах математической физики // Докл. РАН. 1994. Т. 337. № 3. С. 320-322.

[10] Чечкин $\Phi$. А. Об асимптотике решений одного класса нелинейных эллиптических уравнений в цилиндрической области // УМН. 1994. Т. 49. №4. С. 181-182.

[11] Kondratiev V. A., Veron L. Asymptotic homogeneisation of solution of some nonlinear parabolic and elliptic equations. Preprint № 76/94. France: Univ. de Tours, 1994.

[12] Антонцев С. Н. Локализация решений вырождаюшихся уравнений механики сплошной среды. Новосибирск: Ин-т гидродинамики СО АН СССР, 1986.

[13] Чистяков В.В. О свойствах решений полулинейных параболических уравнений второго порядка // Тр. семинара И. Г. Петровского. 1991. Т. 15. С. 70-107.

[14] Гилбарг Д., Трудингер Н. Эллиптические дифференциальные уравнения с частными производными второго порядка. М.: Наука, 1989.

[15] Ладыженская О. А., Солонников В. А., Уральцева Н. Н. Линейные и квазилинейные уравнения параболического типа. М.: Наука, 1967. 